decreased need for others. The tortoise, although not a mammal, provides the perfect example: its extraordinary defensive shell reduces the need to flee or fight to virtually zero.

Furthermore, the possibility arises that future gene therapies may have serious untoward consequences (Watson \& Andrews, 2002). The genes associated with social defeat may encourage a battered wife to yield to her brutal husband, albeit at the price of chronic depression. Should her relevant genes be deleted by future gene therapy, potential consequences include being killed as a result of not yielding, killing her abuser in retaliation or at least a heightened state of domestic abuse. The potential for such untoward consequences must be considered.

Cantor, C. (2005) Evolution and Posttraumatic Stress: Disorders of Vigilance and Defence. Hove: Routledge.

Gilbert, P. (1992) Depression: The Evolution of Powerlessness. Hove: Lawrence Erlbaum.

Selton, J. P. \& Cantor-Graae, E. (2005) Social defeat risk factor for schizophrenia? British journal of Psychiatry, 187, $101-102$

Sloman, L. (2000) How the involuntary defeat strategy relates to depression. In Subordination and Defeat: An Evolutionary Approach to Mood Disorders and Their Therapy (eds L. Sloman \& P. Gilbert), pp. 47-67. Mahwah, NJ: Lawrence Erlbaum.

Stevens, A. \& Price, J. (2000) Prophets, Cults and Madness. London: Duckworth.

Watson, P. J. \& Andrews, P.W. (2002) Toward a revised revolutionary adaptationist analysis of depression: the social navigation hypothesis. Journal of Affective Disorders, 72, $1-14$

C. Cantor Department of Psychiatry, University of Queensland 4072, Australia.

E-mail: cantor98@powerup.com.au

Authors' reply: We thank Drs Price and Cantor for their interesting reactions. We welcome the prophet as a much more colourful 'endophenotype' for schizophrenia than a dysfunction in eye-tracking or prepulse inhibition. However, whether the increased reproductive fitness of a small number of prophets compensates for the reproductive impairments in patients with schizophrenia and their lonely schizotypal relatives remains to be demonstrated.

E. Cantor-Graae Department of Community Medicine, Lund University, University Hospital UMAS, Malmö, Sweden

J.-P. Selten Department of Psychiatry, University Hospital, PO Box 85500, 3508 GA Utrecht,

The Netherlands. E-mail: jp.selten@azu.nl

\section{Schizophrenia, cancer and imprinting: early nutritional influences}

We read with interest the important findings of Goldacre et al (2005) on the association between schizophrenia and cancer morbidity. Compared with the general population, they found a reduced rate of cancer of the colon in the schizophrenia cohort (adjusted rate ratio $0.72,95 \% \mathrm{CI}$ $0.50-1.01)$ with a trend towards significance $(P=0.06)$. Rates of rectal cancer were significantly reduced in people with schizophrenia (rate ratio $0.57,95 \% \mathrm{CI}$ 0.33-0.93, $P=0.03)$. In their discussion, they emphasised the reduced rate of skin cancer in the schizophrenia cohort (rate ratio $0.56,95 \%$ CI $0.36-0.83, P=0.004$ ).

Recent studies suggest that abnormal insulin-like growth factor-2 (IGF-2) imprinting is aetiological in the development of colorectal cancer (Jirtle, 2004). Genomic imprinting occurs following epigenetic modification of the germ line, which results in parent-of-origin dependent, monoallelic gene expression in somatic cells. Epigenetic changes in the genome are stable but reversible alterations in a $\mathrm{CpG}$ dinucleotide or histones, for example through changes in methylation. The genome of colonic epithelium from patients with colorectal cancer is hypomethylated compared with normal colonic epithelia (Feinberg \& Vogelstein, 1983). Hypomethylation results in the loss of IGF-2 imprinting. We proposed abnormal imprinting (deletion of paternally expressed IGF-2) as a possible mechanism associated with schizophrenia risk (Abel, 2004). Early nutritional influences (prenatal/maternal) may stimulate changes in cytosine methylation to which imprinted genes such as IGF-2 seem susceptible. Early nutrition may influence susceptibility not only to adult obesity, diabetes and cardiovascular disease (Waterland \& Jirtle, 2004) but also to schizophrenia. This suggests that early nutritional interventions aimed at preventing chronic disease are an exciting possibility in schizophrenia. This view is supported by Dutch and more recent Chinese data which indicated that rates of schizophrenia doubled following prenatal exposure to famine (St Clair et al, 2005).

Abel, K. M. (2004) Foetal origins of schizophrenia: testable hypotheses of genetic and environmental influences. British Journal of Psychiatry, 184, 383-385.

\section{Feinberg, A. P. \& Vogelstein, B. (1983)}

Hypomethylation distinguishes genes of some human cancers from their normal counterparts. Nature, 30I 89-92.
Goldacre, M. J., Kurina, L. M., Wotton, C. J., et al (2005) Schizophrenia and cancer: an epidemiological study. British Journal of Psychiatry, 187, 334-338.

Jirtle, R. L. (2004) IGF2 loss of imprinting: a potentia heritable risk factor for colorectal cancer. Gastroenterology, 126, I190-120I.

St Clair, D., Xu, M., Wang, P., et al (2005) Rates of adult schizophrenia following prenatal exposure to the Chinese famine of 1959-1961. JAMA, 294, 557-562.

Waterland, R. A. \& Jirtle, R. L. (2004) Early nutrition, epigenetic changes at transposons and imprinted genes, and enhanced susceptibility to adult chronic diseases. Nutrition, 20, 63-68.

K. M. Abel Centre for Women's Mental Health Research, University of Manchester, Oxford Road, Manchester MI3 9PL, UK.

E-mail: Kathryn.M.Abel@manchester.ac.uk

M. P. Allin Division of Psychological Medicine, Institute of Psychiatry, London, UK

R. L. Jirtle Department of Radiation Oncology, Duke University, Durham, North Carolina, USA

\section{Post-traumatic stress disorder after life events}

In their interesting article Mol et al (2005), who conclude that life events might cause as many post-traumatic stress disorder (PTSD) symptoms as do traumatic events. The design and conclusions of the study raise some doubts.

Our first concern relates to the assumption that a particular symptom or disorder may be diagnosed in the absence of the fundamental nature or essence of the disorder. The authors claim to identify PTSD in the absence of (a) a traumatic event and (b) a response involving intense fear, helplessness or horror. Both these criteria are diagnostic A criteria of the DSM-IV and essential features of PTSD. Diagnosing PTSD with disregard for a traumatic event is like diagnosing a major depressive disorder in the absence of depressed mood.

Our assumption regarding the broad use of the PTSD diagnosis is strengthened by the instrument used for assessing PTSD symptoms, which has high sensitivity but lacks specificity (Carlson, 1997). In addition, it is questionable whether a self-report scale can assess the clinical relevance of symptoms. Our concern is corroborated by the results in Table 4 . The only item on which the life events group scored higher than the traumatic events group was the non-specific symptom of 'having trouble concentrating', whereas the traumatic events group scored higher on 'trauma-specific items' such as amnesia and hyperarousal. 